\title{
CÍRCULO DE SEGMENTACIÓN DEL SISTEMA EDUCATIVO CHILENO: DESTINO LABORAL DE EGRESADOS DE PEDAGOGÍA EN EDUCACIÓN BÁSICA
}

\section{Algunos elementos contextuales y conceptuales}

Las teorías de la reproducción, como críticas al ideario meritocrático, atribuyen a la escuela una función de reproductora de las desigualdades sociales en las sociedades de clases. Bourdieu y Passeron (2003) enfatizan el rol de los elementos simbólicos en la reproducción de la desigual estructura social, y de la escuela en particular, dada su condición de sistema simbólico fundamental, encargado de la transmisión de la cultura legitimada, la de las elites, operando una suerte de inculcación cultural e ideológica que perpetúa las relaciones de poder entre las clases sociales.

Entonces, la escuela, al inculcar el "habitus"3 de la clase dominante, genera la situación de desventaja cultural de los estudiantes de clases populares, relegándolos a su posición social originaria y contribuyendo con ello a la reproducción social.

Esta idea de reproducción a manos de la escuela ha sido extrapolada al sistema educativo y particularmente al sistema de educación superior, que en el caso chileno es extensamente analizada por Brunner (2005) y una serie de documentos posteriores que describen al sistema en un contexto de "avanzada privatización e intensa 'mercadización"'.

Señala Brunner que la masificación en el acceso a la educación superior ha generado la fuerte estratificación socioeconómica de las carreras, dado que la selección académica de los estudiantes se homologa a un proceso de selección social, hecho que se demuestra

3 Entendido como el corpus de disposiciones que recogen la identidad e historia sobre el cual se estructuran la lógica interna y la dinámica operativa de un grupo social. 
con claridad en la distribución de los puntajes de ingreso, los cuales, a su vez, se asocian indisolublemente al ingreso familiar de los estudiantes y a sus trayectorias escolares previas. Éstas, circularmente, están fuertemente determinadas por el estatus socioeconómico del hogar de proveniencia (Brunner, 2006; OECD, 2004).

De este modo, tal como lo señalasen Bourdieu y Passeron (2003), los postulantes con mayor capital cultural transmitido por el hogar, que además cuentan con mayor capital escolar acumulado en su trayectoria, son los que acceden a las universidades de mayor reputación (en calidad de "insumos" para esa misma reputación en la que certificarán haber sido formados), capitalizando de ese modo una nueva ventaja que facilita su acceso al mercado laboral y una movilidad "patrocinada" (Turner, 1961) hacia ocupaciones, a su vez, de mayor reputación, estratificándose así también el mercado ocupacional de destino.

En este escenario, señala Brunner (2005), las instituciones de educación superior operan bajo una lógica de competencia por estudiantes, recursos (humanos y financieros) y reputaciones. La reputación es fuertemente determinada por las "dotaciones" de las instituciones de educación superior, constituida por su capital histórico (subsidios e ingresos acumulados), humano (personal académico, administrativo y de apoyo), social (identidad dada por la dinámica de relaciones y normas y tipo de vínculos externos) y reputacional (prestigio de sus académicos y funcionamiento).

Desde esta perspectiva, las universidades de data anterior a la aparición del sistema de mercado en la educación superior (universidades tradicionales) cuentan con una ventaja competitiva inicial en comparación con aquellas que las siguen reputacionalmente, las cuales dependen fuertemente de su capital humano y social para aspirar a grados crecientes de mejor reputación, basada en criterios de prestigio social y académico de sus académicos y egresados, el desarrollo de sus áreas de conocimiento, la calidad del servicio ofrecido, el crecimiento de su capital social y el éxito de la gestión económica. 
22 CÍRCULO DE SEGMENTACiÓN DEL SISTEMA EDUCATIVO CHILENO - Andrea Ruffinelli, Alexis Guerrero

Este contexto de competencia por estudiantes describe tres fenómenos que caracterizan actualmente al sistema de educación superior chileno: (1) una expansión segmentada del mercado mediante la creación de nuevas vacantes, programas y sedes; (2) una estratificación escalonada del mercado según el nivel de selectividad académica y social que logra tener cada entidad, y (3) una diferenciación del valor económico y de prestigio de los títulos profesionales emitidos por las distintas entidades.

El mismo Brunner proponía en 2005 una tipología de instituciones universitarias que intentaba dar cuenta de los efectos de las dinámicas del mercado en el contexto de la política pública de los últimos lustros y de su combinación con la historia de las instituciones. Señalaba que dicha tipología permitía, además, identificar las funciones sociales que las instituciones cumplen y se estructuraba sobre la base de cuatro principios: (1) distinguir entre entidades públicas y privadas; (2) clasificar según nivel de selectividad; (3) determinar la función social de cada categoría, y (4) incorporar un amplio rango de variables de clasificación con el fin de dar cuenta de la heterogeneidad.

A inicios de 2009, el mismo Brunner publica una nueva versión de esta tipología, a partir del debate sobre universidades "cota mil" que regresa a la discusión la forma de caracterizar y clasificar a las instituciones universitarias. Menciona que las categorías empleadas por la estadística internacional ${ }^{4}$, basadas en la distinción pública y privada más financiamiento, resultan útiles sólo para efectos administrativos y estadísticos, no ofrecen una "clasificación suficiente de las universidades chilenas ni sirven tampoco, por lo mismo, para distinguir diversos tipos entre ellas que permitan agruparlas, identificar sus características comunes y diferencias específicas".

En este escenario, particularmente desde la perspectiva de la formación inicial docente, se observa un creciente atractivo de las carreras de pedagogía que responde, por un lado, al proceso que caracteriza transversalmente al sistema de educación superior, el de

4 Clasificación y definiciones tomadas de UNESCO, Instituto de Estadísticas, Glosario. Disponible en: http://www.uis.unesco.org/glossary/index.aspx?lang=es 
su masificación en el acceso, fenómeno que se aprecia con especial fuerza en las carreras de pedagogía, las que han incrementado sus matrículas en una proporción superior al promedio de crecimiento del conjunto del estudiantado 5 .

Particularmente en el caso de pedagogía básica el incremento en la matrícula se asocia con el surgimiento y/o evolución de un significativo grupo de programas académicos, dependientes de un número también significativo de universidades públicas y privadas que han encontrado un singular y rentable nicho en la oferta de carreras que denominan "regulares".

Estas "nuevas carreras" regulares reciben tal denominación amparadas en la desregulación que caracteriza al sistema, pese a exigir mínimos requisitos de ingreso (excluyendo particularmente rendición y puntaje PSU), implementarse bajo modalidades y sobre la base de proyectos curriculares de discutible calidad y con mínimos requisitos de egreso y titulación, en condiciones de considerable menor costo y muchas veces en horario y condiciones flexibles, orientadas a recién egresados de enseñanza media de los quintiles de ingreso más bajos y a trabajadores, apelando la democratización en el acceso a la educación superior y apostando al sueño de movilidad social de grupos históricamente marginados. De este modo, universidades privadas, públicas e institutos profesionales imparten en Chile la carrera de pedagogía en educación básica.

Tras el descenso que se observara a inicios de los 90, en los últimos años la opción por carreras de pedagogía se ha elevado progresivamente, llegando a situarse entre las primeras preferencias de los postulantes ${ }^{6}$, situación que encuentra explicación en al menos tres factores: (a) la implementación de una serie de políticas y beneficios orientados al fortalecimiento de la profesión docente y el mejoramiento de sus condiciones de trabajo (incluyendo estabilidad

5 El mayor crecimiento se observa en la matrícula en universidades privadas, en carreras de Educación Básica y Educación Física, y la oferta de vacantes para carreras de pedagogía creció en mayor proporción en las universidades privadas en los últimos años.

6 La matrícula total en carreras de educación aumentó, entre 1994 y 2004, de 24.973 a 67.015 estudiantes. 
laboral, existencia de un débil sistema de evaluación docente e incrementos salariales disociados del desempeño); (b) el incremento de alternativas de financiamiento orientadas específicamente a los sectores más desfavorecidos, y (c) la alta empleabilidad de los egresados.

Por otra parte, dos estudios realizados en 2005 y 2007 acerca de la oferta de programas especiales de pedagogía básica confirman la incapacidad de la mayoría de dichas propuestas y su implementación ${ }^{7}$ para responder a las necesidades de formación inicial docente de calidad que el país necesita. En ambos estudios se señala que no es posible, con la información disponible, emitir juicios fundados acerca de la capacidad de estos programas regulares para responder eficaz y eficientemente a la necesidad de docentes de calidad en Chile, y mucho menos hacer distinciones entre programas que se acercan con mayor o menor éxito a tal objetivo.

En suma, como señala Brunner (2005), estaríamos en presencia de un sistema básicamente reputacional, en el que las universidades de más alta selectividad (basadas en su prestigio y condiciones del servicio ofrecido) incorporan activamente al estudiante entre las variables críticas que determinan finalmente su reputación, en lo que se ha denominado "tecnología del insumo-cliente" (Rothschild y White, 2003 en Brunner, 2006), y que consiste en que los compradores del servicio educativo aportan simultáneamente a la institución un insumo básico para la cadena productiva, cual es el "efecto pares", referido a que los resultados no dependen exclusivamente de las características individuales sino además del contexto (Cox, 2002), constituyendo una consecuencia potencialmente importante, tanto para mejorar los resultados de los estudiantes más desfavorecidos como para aminorar los resultados de los estudiantes más aventajados (Angrist y Lang (2004).

Es así como la reputación de la institución -dada por el prestigio de sus académicos, la selectividad de estudiantes y el prestigio de sus

7 Así como la de un considerable grupo de programas incluidos en los estudios por ser teóricamente pertenecientes a la modalidad concurrente, pero que finalmente comparten muchas de sus características con los de la modalidad especial. 
egresados- es transmitida mediante las credenciales de sus egresados, las que, por ser de diferente tipo y nivel, cuentan con distintos valores de intercambio para obtener ocupaciones (Davis, 1992 en Brunner, 2006).

Un estudio de Rappaport y colaboradores (2004) muestra una importante variación en el nivel de ingresos de profesionales de una misma carrera egresados de distintas universidades. Si bien el estudio analiza los ingresos para egresados de seis carreras, entre las que no se cuenta educación básica, de todos modos permite señalar como razonable que una porción considerable de tal diferencia pueda ser atribuible a la reputación y selectividad de las diferentes entidades, y que la credencial en sí constituye una señal vital para la toma de decisiones respecto de clasificación y selección de postulantes a puestos de trabajo de diferente jerarquía (Brunner, 2006).

A la luz de este contexto se desarrolla el estudio en que se basa el presente informe, cuyo objetivo fue analizar el destino laboral de los egresados de pedagogía en educación básica de instituciones de educación superior chilenas, a partir del origen educativo y de la eventual relación con el tipo de institución de educación superior de egreso.

La indagación se realizó mediante una encuesta aplicada a 246 egresados y/o titulados entre 2006 y 2007 de la carrera de pedagogía en educación básica en 17 instituciones de educación superior chilenas, que incluyen la presencia de al menos una entidad por cada tipo de institución considerada en la tipología de universidades chilenas (Brunner, 2005, 2009).

Esta cifra de 246 corresponde al 14,5\% de la totalidad de egresados y/o titulados para el periodo, informados por las instituciones de educación superior consideradas. Es preciso consignar la dificultad con que este tipo de estudios se encuentra para establecer contacto con los sujetos de interés, a partir de la obtención de unos datos que deben ser entregados voluntariamente por las instituciones de formación inicial, hecho que constituye una primera restricción a una mayor cobertura de la población en estudio. A su vez, con 
frecuencia esos datos pierden vigencia tras el egreso, hecho que constituye una segunda restricción a la cobertura de la población en estudio, ello sumado a la dificultad para establecer contacto efectivo y lograr disposición para responder al instrumento, lo que que configura una tercera restricción a las posibilidades de ampliar el número de casos en estudio.

Tomando en cuenta las consideraciones señaladas, así como el número de casos estudiados y su forma de reclutamiento -ajustada a las posibilidades efectivas y distinta de un muestreo representativo-, no es posible generalizar las conclusiones del estudio al universo de egresados de la carrera de pedagogía en educación básica en Chile; no obstante, se estima que se trata de conclusiones que resultan significativas para el análisis de esta realidad en el país.

En el estudio se analiza fundamentalmente el itinerario de los egresados desde la dependencia administrativa del establecimiento educativo de origen, pasando por el tipo de institución de educación superior en que cursan sus estudios de pedagogía básica, hasta llegar a la ocupación actual, considerando la dependencia administrativa del establecimiento educativo en que laboran y las condiciones en que desempeñan su trabajo.

Para posibilitar los análisis descritos fue preciso generar una tipología funcional a los objetivos del estudio, de manera que el número de casos en cada categoría permitiera efectivamente realizar los análisis previstos. Esta tipología es más simple que la propuesta por Brunner pero se basa en ella, se elabora sobre todo a partir de criterios de administración y selectividad (asociado este último criterio a indicadores de prestigio) y permite definir cuatro tipos de instituciones: públicas selectivas, públicas de selectividad baja o nula, privadas selectivas y privadas de selectividad baja o nula. A la categoría de instituciones privadas de selectividad baja o nula se agrega instituto profesional, dado que se contó con la participación de un grupo de egresados de dicha procedencia, asumiéndose la similitud de este tipo de entidades con las características propias de las instituciones privadas de baja o nula selectividad. 
Tabla 1: Composición del grupo de casos

\begin{tabular}{|c|c|c|c|c|}
\hline \begin{tabular}{|l} 
Tipo de \\
institución
\end{tabular} & \begin{tabular}{|c|} 
Total de egresados \\
$2006-2007$
\end{tabular} & $\begin{array}{l}\text { Titulado o egresado } \\
2006-2007 \text { que } \\
\text { responden encuesta }\end{array}$ & $\begin{array}{l}\% \text { del total de } \\
\text { egresados de esa } \\
\text { institución }\end{array}$ & $\begin{array}{l}\% \text { del total de } \\
\text { encuestados }\end{array}$ \\
\hline $\begin{array}{l}\text { Tipo 1: Públicas } \\
\text { selectivas }\end{array}$ & 400 & 59 & 14,7 & 23,7 \\
\hline PUC & 243 & 29 & 11,9 & 11,8 \\
\hline PUCV & 82 & 13 & 15,8 & 5,3 \\
\hline UDEC & 75 & 17 & 22,6 & 6,6 \\
\hline $\begin{array}{l}\text { Tipo 2: Públicas } \\
\text { baja o nula } \\
\text { selectividad }\end{array}$ & 742 & 93 & 12,5 & 37,8 \\
\hline UMCE & 195 & 47 & 24,1 & 19,1 \\
\hline U BÍO BÍO & 99 & 16 & 16,1 & 6,5 \\
\hline UCM & 95 & 13 & 13,7 & 5,3 \\
\hline UPLA & 311 & 13 & 4,2 & 5,3 \\
\hline U DE LA SERENA & 42 & 4 & 9,5 & 1,6 \\
\hline $\begin{array}{l}\text { Tipo 3: Privadas } \\
\text { selectivas }\end{array}$ & 310 & 53 & 17,1 & 21,7 \\
\hline UDP & 55 & 10 & 18,1 & 4,1 \\
\hline UFT & 25 & 9 & 36 & 3,7 \\
\hline U A. HURTADO & 28 & 8 & 28,6 & 3,3 \\
\hline UANDES & 101 & 13 & 12,9 & 5,3 \\
\hline $\begin{array}{l}\text { Tipo 4: Privadas } \\
\text { baja o nula } \\
\text { selectividad }\end{array}$ & 342 & 54 & 15,8 & 21,9 \\
\hline IP CHILE & 123 & 23 & 18,7 & 9,3 \\
\hline U CENTRAL & 15 & 4 & 26,6 & 1,6 \\
\hline UAHC & 110 & 16 & 14,5 & 6,5 \\
\hline U BOLIVARIANA & 29 & 2 & 6,9 & 0,8 \\
\hline U DEL MAR & 65 & 9 & 13,8 & 3,7 \\
\hline Total & 1693 & 246 & 14,5 & 100 \\
\hline
\end{tabular}

\section{Algunas conclusiones del estudio para iniciar la discusión:}

Los docentes que han egresado de establecimientos educativos particulares pagados y que estudian pedagogía en educación básica lo hacen mayoritariamente en las instituciones de educación superior de mayor prestigio, universidades privadas selectivas y, en segundo término, públicas selectivas, y al egresar retornan a su origen, desempeñándose en establecimientos educativos particulares pagados, en condiciones laborales comparativamente superiores 
28 CÍRCULO DE SEGMENTACIÓN DEL SISTEMA EDUCATIVO CHILENO - Andrea Ruffinelli, Alexis Guerrero

a las de docentes egresados de establecimientos subvencionados e instituciones no selectivas.

Los docentes que han egresado de establecimientos educativos municipales y que estudian pedagogía en educación básica lo hacen mayoritariamente en las instituciones de educación superior de menor prestigio, instituciones educativas públicas y privadas no selectivas, así como en universidades públicas selectivas, y al egresar reproducen también la dependencia de su establecimiento escolar de origen, retornando a desempeñarse laboralmente en escuelas municipales, en condiciones laborales comparativamente inferiores a las de docentes egresados de establecimientos subvencionados e instituciones selectivas.

La concentración de egresados provenientes de los sistemas municipal y particular subvencionado en las carreras de educación básica en instituciones de educación superior no selectivas (públicas y privadas) es casi total, no superándose el $4 \%$ de estudiantes provenientes del sistema particular pagado. En cambio, en las universidades privadas selectivas la concentración de estudiantes provenientes del sistema particular pagado llega, en promedio, casi al 50\%.

Tabla 2. Clasificación de Universidades. Dependencia del establecimiento educativo del que Ud. egresó de enseñanza media

\begin{tabular}{|c|c|c|c|c|c|c|}
\hline & \multicolumn{3}{|c|}{$\begin{array}{c}\text { Dependencia del establecimiento educativo } \\
\text { del que Ud. egresó de enseñanza media }\end{array}$} & \multirow[t]{2}{*}{ Total } \\
\hline & & & Municipal & Part. Subv & Part. Pagado & \\
\hline \multirow{8}{*}{$\begin{array}{l}\text { Clasificación } \\
\text { de } \\
\text { universidades }\end{array}$} & \multirow{2}{*}{ Públ. selectivas } & Recuento & 21 & 24 & 9 & 54 \\
\hline & & $\%$ & $38,9 \%$ & $44,4 \%$ & $16,7 \%$ & $100,0 \%$ \\
\hline & \multirow{2}{*}{$\begin{array}{l}\text { Públ. baja o nula } \\
\text { selectividad }\end{array}$} & Recuento & 35 & 44 & 2 & 81 \\
\hline & & $\%$ & $43,2 \%$ & $54,3 \%$ & $2,5 \%$ & $100,0 \%$ \\
\hline & \multirow{2}{*}{ Priv. selectivas } & Recuento & 6 & 15 & 19 & 40 \\
\hline & & $\%$ & $15,0 \%$ & $37,5 \%$ & $47,5 \%$ & $100,0 \%$ \\
\hline & \multirow{2}{*}{$\begin{array}{l}\text { Priv. baja o nula } \\
\text { selectividad }\end{array}$} & Recuento & 16 & 33 & 2 & 51 \\
\hline & & $\%$ & $31,4 \%$ & $64,7 \%$ & $3,9 \%$ & $100,0 \%$ \\
\hline \multirow{2}{*}{\multicolumn{2}{|c|}{ Total }} & Recuento & 78 & 116 & 32 & 226 \\
\hline & & $\%$ & $34,5 \%$ & $51,3 \%$ & $14,2 \%$ & $100,0 \%$ \\
\hline
\end{tabular}

Cerca del 66\% de los docentes egresados de establecimientos municipales trabaja hoy también en establecimientos educativos municipales; un 63\% de los docentes egresados de establecimientos 
educativos particulares subvencionados lo hace hoy en establecimientos del mismo tipo y cerca de un 58\% de los egresados de establecimientos pagados se desempeña actualmente en establecimientos del mismo tipo.

Ningún docente egresado de establecimiento educativo municipal se desempeña hoy en un establecimiento educativo particular pagado y menos del $10 \%$ de los docentes egresados de establecimientos particulares pagados trabaja actualmente en establecimientos municipales.

Tabla 3: Dependencia laboral actual según dependencia de establecimiento educativo de egreso

\begin{tabular}{|c|c|c|c|c|c|c|}
\hline & \multicolumn{3}{|c|}{ Dependencia egreso } & \multirow{2}{*}{ Total } \\
\hline & & & Municipal & Part. Subv & Part. Pagado & \\
\hline \multirow{6}{*}{$\begin{array}{l}\text { Dependencia } \\
\text { actual }\end{array}$} & \multirow{2}{*}{ Municipal } & Recuento & 27 & 14 & 0 & 41 \\
\hline & & $\%$ & $65,9 \%$ & $34,1 \%$ & $0 \%$ & $100,0 \%$ \\
\hline & \multirow{2}{*}{ Part. Subv } & Recuento & 36 & 72 & 6 & 114 \\
\hline & & $\%$ & $31,6 \%$ & $63,2 \%$ & $5,3 \%$ & $100,0 \%$ \\
\hline & \multirow{2}{*}{ Part. Privado } & Recuento & 3 & 11 & 19 & 33 \\
\hline & & $\%$ & $9,1 \%$ & $33,3 \%$ & $57,6 \%$ & $100,0 \%$ \\
\hline \multirow{2}{*}{\multicolumn{2}{|c|}{ Total }} & Recuento & 66 & 97 & 25 & 188 \\
\hline & & $\%$ & $35,1 \%$ & $51,6 \%$ & $13,3 \%$ & $100,0 \%$ \\
\hline
\end{tabular}

Virtualmente, ninguno de los egresados de instituciones privadas no selectivas se desempeña en establecimientos particulares pagados. Del mismo modo, las universidades privadas selectivas virtualmente no cuentan con egresados desempeñándose en el sistema educativo municipal.

Tabla 4: Dependencia laboral actual según tipo de institución de educación superior de egreso

\begin{tabular}{|c|c|c|c|c|c|c|}
\hline & \multicolumn{3}{|c|}{ Dependencia actual } & \multirow{2}{*}{ Total } \\
\hline & & & Municipal & Part. Subv & Part. Pagado & \\
\hline \multirow{8}{*}{$\begin{array}{l}\text { Clasificación } \\
\text { de } \\
\text { universidades }\end{array}$} & \multirow{2}{*}{ Públ. selectivas } & Recuento & 10 & 26 & 15 & 51 \\
\hline & & $\%$ & $19,6 \%$ & $51,0 \%$ & $29,4 \%$ & $100,0 \%$ \\
\hline & \multirow{2}{*}{$\begin{array}{l}\text { Públ. baja o nula } \\
\text { selectividad }\end{array}$} & Recuento & 22 & 51 & 6 & 79 \\
\hline & & $\%$ & $27,8 \%$ & $64,6 \%$ & $7,6 \%$ & $100,0 \%$ \\
\hline & \multirow{2}{*}{ Priv. selectivas } & Recuento & 1 & 20 & 15 & 36 \\
\hline & & $\%$ & $2,8 \%$ & $55,6 \%$ & $41,7 \%$ & $100,0 \%$ \\
\hline & \multirow{2}{*}{$\begin{array}{l}\text { Priv. baja o nula } \\
\text { selectividad }\end{array}$} & Recuento & 13 & 26 & 1 & 40 \\
\hline & & $\%$ & $32,5 \%$ & $65,0 \%$ & $2,5 \%$ & $100,0 \%$ \\
\hline \multirow{2}{*}{\multicolumn{2}{|c|}{ Total }} & Recuento & 46 & 123 & $2,5 \%$ & $100,0 \%$ \\
\hline & & $\%$ & $22,3 \%$ & $59,7 \%$ & $18,0 \%$ & $100,0 \%$ \\
\hline
\end{tabular}


30 CíRCULO DE SEGMENTACiÓN DEL SISTEMA EDUCATIVO CHILENO - Andrea Ruffinelli, Alexis Guerrero

Estos hallazgos vienen a corroborar antiguas y extendidas impresiones, intuiciones y sentidos comunes, propios de un perfecto reflejo de lo que señalara ya en 2004 el informe de la Organisation for Economic Co-operation and Development (OECD, 2004: 277): que el sistema educativo chileno está "conscientemente estructurado por clases sociales", reproduciendo así la grave desigualdad que vive el país. Afirmaba además que: "La escasez de capacidad docente y supervisora afecta mayoritariamente a los estudiantes de bajos ingresos, que tienen mínimos recursos familiares para manejarse en un sistema educacional que no logra estándares altos. Los estudiantes de bajos ingresos pagan el precio más alto por las falencias del sistema" (OECD, 2004: 290).

Si se asume que, al menos a partir de dicho informe, la segmentación del sistema escolar resulta evidente e ineludible, reconociéndose un "apartheid" educativo compuesto por sistemas escolares cerrados y excluyentes de administración -que en la práctica se homologan al origen socioeconómico de los estudiantes-, es plausible plantear ahora, a partir de nuestros datos, que existe un correlato de este "apartheid" en las entidades formadoras de docentes y en el retorno a los sistemas escolares de origen.

Esta segmentación social en el sistema educativo, generada paralelamente a un proceso incremental de inequidad del sistema, configura un escenario en que se estarían formando docentes explícitamente destinados a alguno o algunos de dichos subsistemas, en una incorporación funcional del sistema de educación superior al círculo reproduccionista, que segrega tanto a estudiantes del sistema escolar como de formación inicial docente según el origen socioeconómico, obligándolos a experimentar separadamente sus procesos formativos para volver luego como docentes al mismo subsistema educativo en el que fueron formados.

Se trata de la segmentación y segregación educativa en su máxima expresión, con una transversalidad supina y superlativa. En suma, la perpetuación en todo nivel de la negación de la posibilidad de mixtura y cohesión social necesaria para corregir inequidades, lograr los aprendizajes necesarios e incorporarse efectivamente a una 
sociedad democrática e inclusiva. Se reproduce así, en todos los niveles del sistema educativo, una realidad caracterizada por una "tremenda segmentación y segregación, en que cada niño, niña y joven se educa con sus pares desde el punto de vista social y económico, negándose en la experiencia escolar un (re)conocimiento de los otros distintos a sí, lo que limita el aprendizaje recíproco entre ellos (efecto pares), que requiere encuentro entre quienes vienen de ambientes más y menos cercanos a la cultura letrada" (García Huidobro, J.E., 2009).

Tal como señala García Huidobro (2009), si "lo que se plantea es que no sólo se debe dar lo mismo a todos, es decir, que la educación sea universal, sino que debe entregarse más a los que poseen mayores carencias y dificultades para asegurar igualdad de oportunidades, entendiendo que asegurar esta igualdad no significa entregar a todos la misma educación, sino que una educación que permita que todas y todos puedan aprender, es decir, entregar igualdad de oportunidades no meramente en el tratamiento educativo (la educación que reciben), sino también en los resultados (los aprendizajes que logran)", es posible comprender que estas constataciones constituyen un lapidario atentado contra la posibilidad de un sistema que permita educarse y educar junto a personas -compañeros de aula y docentes-, que cuenten con un capital social y cultural distinto del propio, permitiendo, por una parte, actuar al "efecto pares" y, por otro, potenciar el proceso educativo en un contexto integrador.

Un escenario contundente que se expresa además en todas las posibles desventajas para los más desventajados desde su origen: para los docentes egresados del sistema particular subvencionado y especialmente para los docentes egresados del sistema municipal. Por otro lado, para los docentes egresados de instituciones de educación superior públicas no selectivas y particularmente privadas no selectivas: los menores índices de empleabilidad, las menores probabilidades de ser directivos o docentes de educación superior, la menor velocidad de inserción laboral, las peores condiciones laborales, las más bajas remuneraciones iniciales, las menores expectativas de alza durante el ejercicio y la garantía de una vida laboral que retornará al mismo ámbito socioeconómico, cultural y educativo del que provienen (Ruffinelli, 2009). 
32 CÍRCULO DE SEGMENTACiÓN DEL SISTEMA EDUCATIVO CHILENO - Andrea Ruffinelli, Alexis Guerrero

Si bien el hecho de acceder a educación superior, especialmente en el caso de los docentes provenientes de estratos socioeconómicos más deprivados, reivindica una realidad histórica de marginación y posibilita la efectiva movilidad social de estos docentes, no es menos cierto que, tal como se señalara recién, ellos han egresado de instituciones de educación superior evidentemente menos selectivas y menos prestigiosas, en las que probablemente han recibido una formación profesional más débil, lo que, asociado a sus propias debilidades académicas de origen sociocultural y educativo, configura un escenario poco propicio para el desarrollo de un profesional idóneo, capaz de revertir la precariedad con que enfrentan el proceso educativo los estudiantes más vulnerables y producir en ellos los aprendizajes necesarios de la manera más eficaz y eficiente.

En este punto parece pertinente señalar la eventual presencia de un círculo vicioso de egresados de educación media que provienen de los estratos y escuelas más vulnerables, que son formados profesionalmente en las instituciones de educación superior de menor selectividad y prestigio y que vuelven a sus mismos nichos de origen, a enseñar, con herramientas probablemente mucho menos potentes, a los hijos de sus amigos de la infancia, a los compañeros de barrio, a los que con mayor urgencia requieren de los mejores docentes, los más preparados. Estos últimos, por otra parte, continúan en su propio círculo, egresando de los colegios de elite, formándose profesionalmente en universidades del mismo tipo y ejerciendo para educar también a los hijos de sus amigos de la infancia, fortaleciendo aún más la preparación para la vida de quienes llegan a ella y se desarrollan en las mejores condiciones, reproduciendo la inequidad social en una traición tautológica, a través de la herramienta más poderosa para, precisamente, disminuirla.

En el estudio se constata que existen diferencias estadísticamente significativas entre las remuneraciones actuales de los docentes según la dependencia de su establecimiento educativo de egreso y el tipo de institución de educación superior de egreso, siendo sustancialmente mayores en el caso de los egresados de establecimientos particulares pagados en comparación con las de egresados de establecimientos municipales y particulares subvencionados, que además son similares 
entre sí. A la vez, se comprueba que son sustancialmente mejores las remuneraciones entre egresados de universidades selectivas, privadas en primer lugar y luego públicas, y sustancialmente más bajas entre egresados de instituciones no selectivas, privadas en primer término y luego públicas.

Además, los docentes egresados de establecimientos particulares pagados y de universidades privadas selectivas se inician laboralmente con mejores remuneraciones y son los que obtienen también las mejoras más sustantivas con el paso del tiempo. En contraste, los docentes egresados de establecimientos municipales y particulares subvencionados y de instituciones de educación superior privadas no selectivas son los que concentran la mayor proporción de egresados en los tramos salariales más bajos y logran también los más bajos incrementos salariales en el tiempo.

- La mitad de los docentes encuestados percibe actualmente ingresos mensuales entre los 351 mil y los 500 mil pesos. Cerca de un 12\% gana menos de $\$ 250.000$. Sólo un $17 \%$ gana más de $\$ 500.000$, y un $3,8 \%$ percibe ingresos por sobre los 650 mil pesos.

Gráfico 1. Ingreso mensual en su actual trabajo

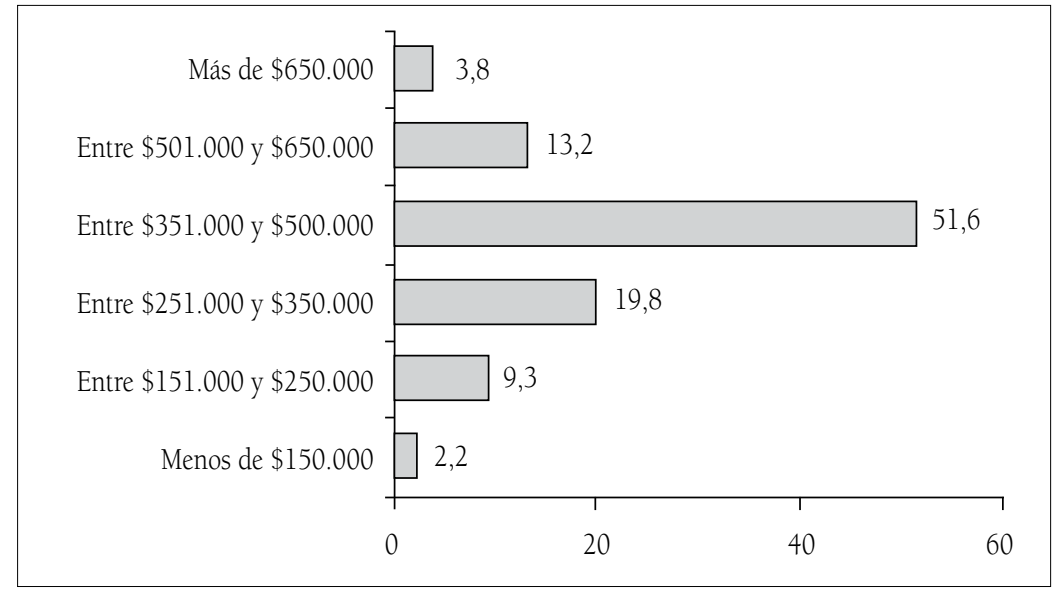

- La mayoría de los egresados de establecimientos particulares subvencionados y municipales (sobre el 75\%) gana entre $\$ 251.000$ y $\$ 500.000$, mientras sólo un 37,5\% de los egresados 
de establecimientos particulares pagados se ubica en ese rango de remuneraciones. La mayoría de los egresados de establecimientos particulares pagados $(54,2 \%)$ gana más de $\$ 500.000$ mensuales. Los egresados de establecimientos municipales y particulares subvencionados muestran niveles similares de remuneraciones mensuales.

Gráfico 2: Ingreso mensual actual según dependencia de establecimiento educativo de egreso

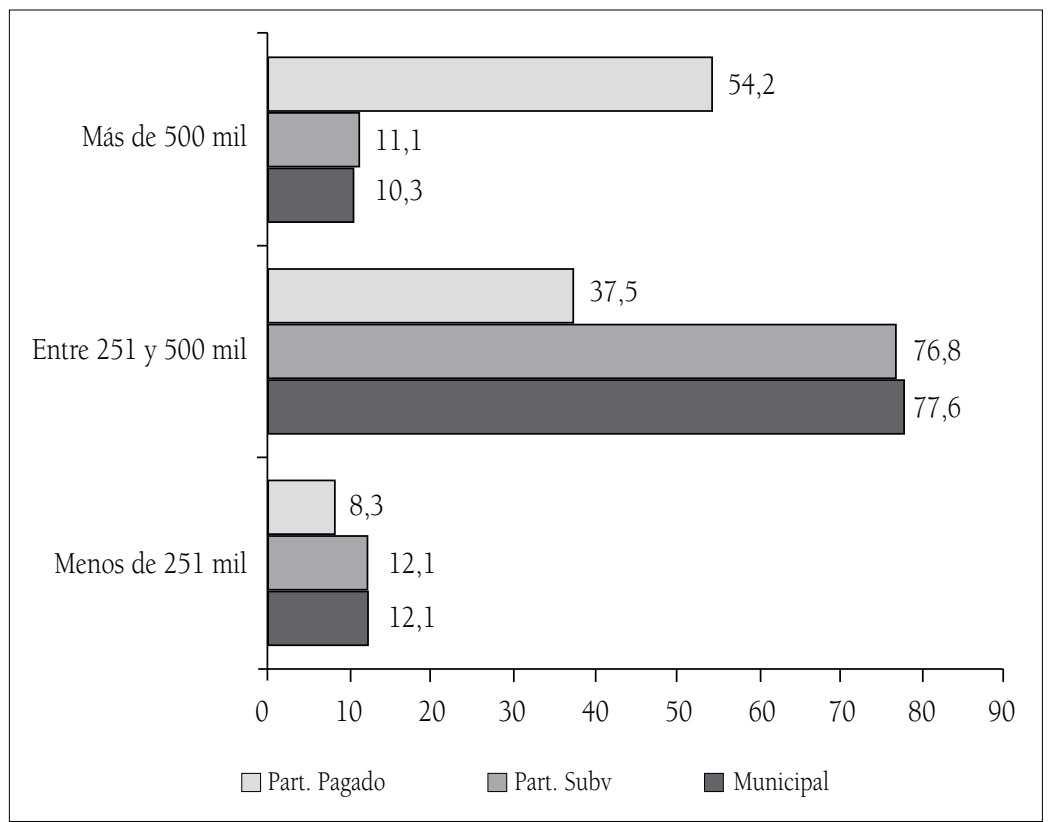

- Los egresados de universidades privadas selectivas concentran la mayor parte de los egresados con ingresos mensuales superiores a $\$ 500.000$ (35,5\%), seguidas de las universidades públicas selectivas. Los egresados con rentas más bajas se concentran entre los egresados de instituciones privadas sin selectividad (21,9\%) y particularmente entre los egresados de instituto profesional: un $54,5 \%$ de estos percibe remuneraciones mensuales inferiores a los $\$ 251.000$ y ninguno declara rentas sobre los $\$ 500.000$. Los egresados de universidades públicas sin selectividad muestran mejores niveles de remuneraciones que los de entidades privadas sin selectividad. 
Gráfico 3: Ingreso mensual actual según institución de educación superior de egreso

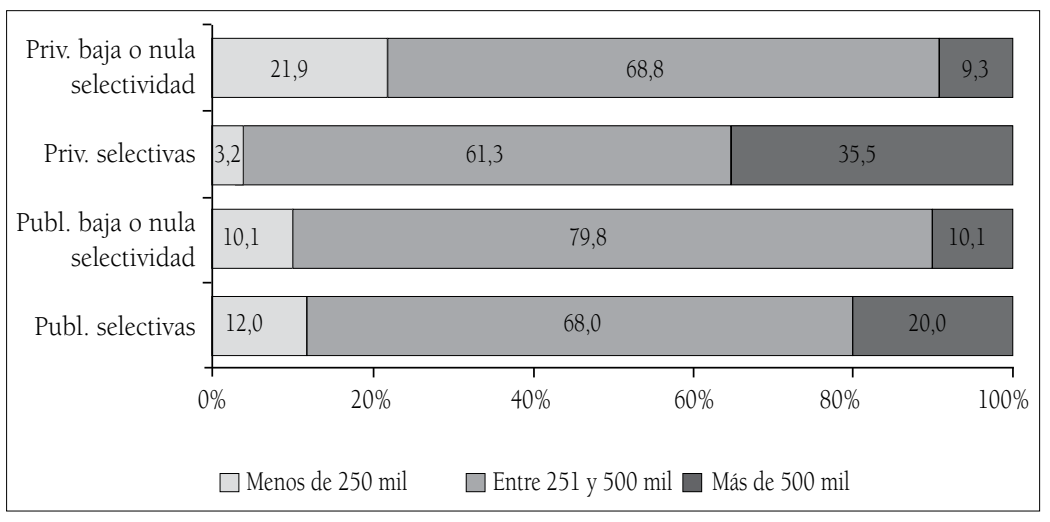

- Mientras los egresados de establecimientos pagados que se ubican en el más alto tramo de remuneraciones tienen un incremento en más de 25 puntos porcentuales entre el primer ingreso y el actual (de 29,6\% a 54,2\%), en el caso de los egresados de establecimientos particulares subvencionados y municipales el incremento entre el primer ingreso y el actual no supera los 5 puntos porcentuales, observándose entre éstos una tendencia a pasar a engrosar el segundo tramo de remuneraciones.

- En su primera remuneración, los egresados de establecimientos particulares subvencionados y municipales triplican a los egresados de establecimientos particulares pagados en el tramo de remuneraciones más bajas. Actualmente, los egresados de establecimientos particulares subvencionados y municipales recién casi han alcanzado los niveles iniciales de ingresos de los egresados de establecimientos pagados. 
36 CÍRCULO DE SEGMENTACIÓN DEL SISTEMA EDUCATIVO CHILENO - Andrea Ruffinelli, Alexis Guerrero

Gráfico 4: Primer ingreso e ingreso actual según dependencia de establecimiento educativo de egreso

\begin{tabular}{|c|c|c|c|c|c|c|}
\hline Part. Priv. ingreso actual & 8,3 & \multicolumn{2}{|l|}{37,5} & \multicolumn{3}{|c|}{54,2} \\
\hline Part. Pag. primer ingreso & 11,1 & \multicolumn{2}{|l|}{59,3} & & \multicolumn{2}{|c|}{29,6} \\
\hline Part. Subv ingreso actual & 12,1 & \multicolumn{3}{|c|}{76,8} & & 1,1 \\
\hline Part Subv. primer ingreso & \multicolumn{2}{|c|}{32,7} & \multicolumn{3}{|c|}{60,6} & 6,7 \\
\hline \multirow{4}{*}{$\begin{array}{l}\text { Municipal ingreso actual } \\
\text { Municipal primer ingreso }\end{array}$} & 12,1 & \multicolumn{3}{|c|}{77,6} & & 0,3 \\
\hline & \multicolumn{2}{|c|}{30,8} & \multicolumn{3}{|c|}{64,6} & 4,6 \\
\hline & $\%$ & $20 \%$ & $40 \%$ & $60 \%$ & $80 \%$ & $100 \%$ \\
\hline & $\square \mathrm{Me}$ & de 250 mil & $\square$ En & $500 \mathrm{mi}$ & de 50 & \\
\hline
\end{tabular}

- Los egresados de universidades privadas selectivas concentran, desde el inicio, la mayor cantidad de docentes con las mejores remuneraciones en el mejor tramo salarial, 27,3\%, y la incrementan en más de ocho puntos porcentuales en la actualidad, llegando a 35,5\%. Los egresados de universidades públicas selectivas parten con una proporción de egresados mucho menor en el tercer y mejor tramo salarial, sin embargo lo incrementan mucho más, en más de 12 puntos porcentuales. Aun así quedan a una distancia de más de 15 puntos porcentuales de la proporción de egresados que en este tramo llegan a tener los egresados de universidades privadas selectivas.

- Los egresados de entidades privadas no selectivas son los que parten con la mayor proporción de egresados en el tramo más bajo de sueldo $(40,5 \%)$ y, pese a que cerca de la mitad de éstos logran avanzar al siguiente con el ingreso actual, siguen siendo el tipo de institución con mayor proporción de egresados en el tramo más bajo de remuneraciones. 
Gráfico 5: Primer ingreso e ingreso actual según tipo de institución de educación superior de egreso

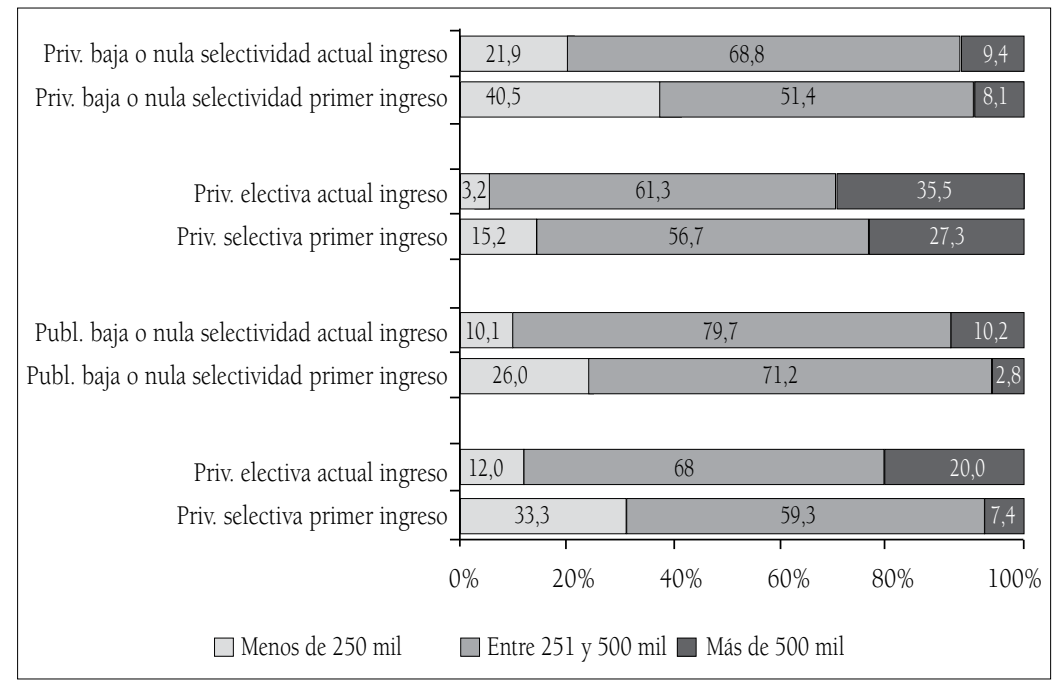

La constatación de remuneraciones fuertemente asociadas al establecimiento de egreso y a la institución de formación inicial constituye una manera particularmente poderosa de expresar la perpetuación de la inequidad mediante la reproducción de las condiciones de origen sociocultural. Alguien podría incluso llamarlo justicia, considerando que obtiene un salario mayor quien ostenta un mejor currículo, que ciertamente incluye el lugar de educación media y superior; sin embargo, enmascara la situación de desigualdad que ha impedido sistemáticamente a los de origen más vulnerable acceder a otras opciones en ambos tipos de educación.

Resulta también posible hipotetizar que el escenario general descrito por los hallazgos del estudio de destino laboral de los egresados de pedagogía básica se configura fundamentalmente a partir de dos hechos: la posibilidad efectiva de elegir, que se encontraría alojada exclusivamente entre los estudiantes de familias que cuentan con capacidad de pago a cambio de formación universitaria; o entre los estudiantes con altos niveles de rendimiento académico (que suelen ser los mismos anteriores y, en menor medida, algunos estudiantes provenientes de sectores con menos recursos, muy resilientes, que sortean con éxito sus condiciones ligadas al origen, quienes logran 
38 CÍRCULO DE SEGMENTACiÓN DEL SISTEMA EDUCATIVO CHILENO - Andrea Ruffinelli, Alexis Guerrero

obtener financiamiento o beca para estudios superiores; por otro lado, la existencia de un ethos, un conjunto de valores y significados propio de los estratos socioeconómicos aventajados que, desde su "habitus", los impulsa a reunirse con sus pares, a ubicarse en los espacios, en los "campos", que las representaciones sociales colectivas y las propias indican que les corresponden por origen.

Se privilegia de este modo una continuidad en las propias redes sociales, en una extensión de la segmentación del sistema escolar, y, en el caso de los estratos más populares, un ethos que los impulsa también a reunirse con sus pares, en otros espacios, por cuestiones culturales, pero apremiantemente también por cuestiones económicas y académicas.

Para un egresado de los estratos medios y bajos, que en rigor se asocia a establecimientos educativos municipales y subvencionados, ciertamente será opción la universidad pública, desde su ethos, pero será selectiva sólo si su rendimiento y economía lo permiten; si no es selectiva, será opción en tanto pueda obtener algún financiamiento acorde con su presupuesto, posibilidad que también se vincula finalmente a cuestiones académicas, ya que de por medio hay exigencias mínimas de rendimiento. De hecho, la opción por universidades públicas selectivas entre egresados de la educación municipal en el último tiempo encuentra gran parte de su explicación en la generación de nuevas e importantes fuentes de financiamiento (becas y créditos) dirigidas a dicho sector, estimulando el acceso de los que logran algunos mínimos, en el caso de crédito, y de los mejores rendimientos, en el caso de las becas.

Asimismo, por cuestiones también económicas y académicas -que van finalmente definiendo un ethos-, las entidades de educación superior privadas no selectivas se han ido configurando en el destino universitario posible para los egresados de los establecimientos municipales, para los más pobres, ante la exigencia social de credenciales académicas. Se trata de una carrera que exige el más bajo puntaje de ingreso en estas instituciones o bien no lo exige; incluso, no es requisito en muchas de ellas la rendición de PSU. Se trata a la vez de una alternativa económica que, si bien en la mayor parte de los casos 
no ofrece acceso a financiamiento, exige aranceles significativamente más bajos en algunos casos y su flexibilidad permite simultáneamente estudiar y trabajar, características que permiten omitir precisamente las variables que imposibilitan el acceso a las entidades selectivas. Aunque muy probablemente estos estudiantes no estén haciendo uso de una opción, porque efectivamente no la tienen, sino más bien de una oportunidad, la única que tienen.

Todo esto, sin considerar la ausencia de criterios objetivos, compartidos y confiables, y mucho menos de dominio público, para llegar a discernir entre la calidad de la formación inicial docente en una u otra entidad ${ }^{8}$.

En tanto, para un egresado del sistema particular pagado la posibilidad de optar es una realidad: puede elegir entre universidades de prestigio, públicas o privadas, entre la tradición y la academia, o sus redes sociales que también le van ofreciendo la academia.

Pareciera que la premisa histórica básica referida a una generalizada primera opción de la mayoría de los eventuales estudiantes de educación superior por las universidades públicas selectivas mantiene una fuerza, pero pierde generalidad en el caso de potenciales estudiantes de estratos socioeconómicos altos, quienes ya disponen de alternativas privadas en el mercado, con niveles de prestigio académico equiparables a los de algunas universidades tradicionales, contando en dicha alternativa, además, con una seguridad plena de extender y fortalecer lazos entre los de su misma clase.

En este escenario, los estudiantes de los estratos medios y bajos, que en rigor se asocian con establecimientos educativos municipales y subvencionados, tienen como única opción las entidades públicas selectivas, en el caso de evidenciar las capacidades académicas que lo hagan merecedor de algún tipo de financiamiento o beca, o bien entidades públicas no selectivas, en las que el monto del arancel es más acorde con sus posibilidades económicas, aunque esto implique

8 Una muestra de un sistema desregulado que confunde y afecta, en definitiva, la libertad de elección, cuando la hay, y cuando es restringida también. Pero eso será tema de otros análisis. 
40 CíRCULO DE SEGMENTACiÓN DEL SISTEMA EDUCATIVO CHILENO - Andrea Ruffinelli, Alexis Guerrero

una eventual renuncia a ingresar en una institución de prestigio académico.

Algo de esto empieza a cambiar por el incremento significativo de alternativas de financiamiento para la educación superior que veníamos señalando, situación que por primera vez hace posible no sólo el acceso para los estratos más vulnerables, sino que abre esa posibilidad a las instituciones más prestigiosas. Sin embargo, esto se cruza con el explosivo crecimiento de instituciones de educación superior no selectivas y de bajo costo, que constituyen la verdadera oportunidad para la mayoría de los egresados de educación media provenientes de estratos populares, considerando que, dado su origen, tipo de capital cultural, condiciones de vida, tipo de educación recibida, nivel de provecho que pueden hacer de ella y competencias que llegan a alcanzar durante su recorrido escolar, difícilmente pueden aspirar a ingresar a universidades prestigiosas y selectivas, compitiendo con egresados que han tenido acceso a las mejores opciones educativas en las mejores condiciones. Son entonces estos últimos los que se van quedando con los mejores salarios, en un círculo segregacionista.

Sin embargo, todo parece indicar que en el mediano plazo esta relación tan claramente lineal entre mayor nivel socioeconómico y acceso a universidades privadas selectivas comenzaría a diluirse, en el contexto del surgimiento de variadas alternativas de financiamiento que se extienden rápidamente hacia los sectores más desfavorecidos, produciendo un viraje significativo hacia la democratización del acceso a la educación superior, e incluso, podría agregarse, a la educación superior de mayor calidad académica (dado que su asignación exige algunos argumentos en este sentido para hacerse efectiva).

Con todo, este intento de corregir la desigualdad histórica vía mejoramiento del sistema de becas y créditos para la educación superior parece mostrar finalmente una débil capacidad correctiva, al constituir opción sólo para aquellos que ya han logrado sortear las múltiples desigualdades asociadas con su origen, y, pese a ellas, han demostrado capacidades para acceder a instituciones de educación superior más prestigiosas y selectivas. 
Sin duda este nuevo escenario requerirá una profunda y detenida observación en el tiempo, con el fin de develar si lo que emerge como un aporte sustantivo a la superación de la inequidad no se constituye finalmente en un entrampe social que replica las desigualdades inyectando recursos diferenciados, provocando un efecto perverso de discriminación positiva que termine por generar ghettos de educación superior privada segregados para pobres, con altos rendimientos en un caso y bajos rendimientos para otro.

Resulta de especial interés en este punto observar la existencia de dos grupos marcadamente distintos en su composición al interior de la categoría de universidades privadas selectivas: uno formado por universidades cuya concentración de estudiantes de educación básica provenientes del sistema particular pagado llega a casi el 85\% en un caso y casi al 70\% en otro, y otro grupo en que la composición de los estudiantes de la carrera se inclina hacia el lado opuesto, con no más de un $12 \%$ de estudiantes provenientes del sistema particular pagado (cifra que se va acercando a la efectiva proporción de dicho subsistema en el sistema educativo total).

Esta es una clara muestra de un efecto de las nuevas alternativas de financiamiento a la educación superior que afecta drásticamente la composición del estudiantado incorporando mixtura social, aunque hace surgir la necesidad urgente de observar longitudinalmente el hecho, dado que, eventualmente, podría implicar un viraje del ethos, del conjunto de valores y significados propio del grupo social aludido, a causa del cambio radical en la composición del estudiantado de un plantel educativo, perdiendo finalmente mixtura y posicionándose como alternativa similar a las instituciones de educación superior privadas no selectivas. En estas últimas, la diferencia estaría dada por los niveles de rendimiento escolar dentro de un mismo estrato socioeconómico, una discriminación intraestrato, generando los mencionados ghettos de educación superior privada para estudiantes pobres de buen rendimiento, que se sumarían a los ghettos de educación superior que ya se han venido configurando para los estudiantes de menores recursos con bajo rendimiento y a los de estudiantes ricos, ambos ya consolidados. 
Ante este panorama, se hace evidente la necesidad de afinar el discurso, generar más y mejores conversaciones, creando condiciones para destrabar nudos estructurales del sistema de educación superior chileno, con el fin de prever y actuar frente a situaciones como las que se describen.

Sería el momento de pensar de verdad en la equidad y, probablemente, la única forma de abordarla con seriedad sería desde una regulación que se logre a partir de una estructura organizacional que efectivamente represente el actual escenario nacional de la educación superior, utilizando como criterio básico estándares mínimos de calidad, que operativamente podrían estar dados por la acreditación institucional, tal como han señalado reiteradamente una multiplicidad de actores. Desde esta nueva estructura básica integradora sería conveniente repensar las regulaciones, siempre desde una perspectiva de aseguramiento de la calidad, con equidad.

Y en este ejercicio no podrán quedar fuera los análisis y decisiones necesarias acerca del sistema único de ingreso a la educación superior, los requisitos mínimos de ingreso, las razones para determinar diferencias entre los mínimos exigidos a las carreras de pedagogía y las otras (especialmente pedagogía básica que ostenta el triste récord de mínima exigencia, cuando la hay), la alternativa de exigencia de cuotas de estudiantes de estratos socioeconómicos desfavorecidos a las universidades (aunque no soluciona la necesidad de mixtura social en las entidades menos prestigiosas) y el necesario análisis acerca del rol de la carrera de educación básica en las instituciones de educación superior y de los docentes en nuestra sociedad, un sinceramiento que podría conducirnos a cuestionar los argumentos de democratización en el acceso, en el caso de una carrera de influencia tan decisiva y fundamental en la población como pedagogía básica.

Urge el debate, urge la acción, urge una sociedad más justa, una educación más equitativa, una mirada más inclusiva en un marco de voluntad política, organización y decisión. 


\section{Referencias bibliográficas}

Angrist, J. y Lang, K. (2002) How Important are Classroom Peer Effects? Evidence from Boston's Metco Program. NBER, Working Paper No 9263.

Bourdieu, P. y Passeron, J.C. (2003) Los herederos. Los estudiantes y la cultura. México: Siglo XXI.

Brunner, J.J.; Elacqua, G.; Tillett, A.; Bonnefoy, J.; González, S.; Pacheco, P. y Salazar, F. (2005) Guiar el mercado. Informe sobre la educación superior en Chile. Santiago de Chile: Universidad Adolfo Ibáñez. Disponible en http://mt.educarchile.cl/mt/jjbrunner/archives/2005/08/ nuevo_libro.html

Brunner, J.J. (2006) Sistema privatizado y mercados universitarios: competencia reputacional y sus efectos. Disponible en www.educarchile.cl

Brunner, J.J. (2009) Tipología y características de las universidades chilenas. Documento para comentarios. Programa Anillo (SOC-01) sobre Políticas de Educación Superior, apoyado por CONICYT, con el patrocinio de la Cátedra UNESCO en Políticas Comparadas de Educación Superior con sede en la Universidad Diego Portales. Disponible en http://mt.educarchile.cl/MT/jjbrunner/archives/ Tipol\%26Caract_080209.pdf

Cox, C. (editor) (2002) Políticas educacionales en el cambio de siglo. La reforma del sistema escolar de Chile. Santiago de Chile: Editorial Universitaria.

García Huidobro, J.E. (2009) Institucionalidad educativa chilena. Análisis desde una perspectiva democrática. Santiago de Chile: Notas para exposición en Escuela de Verano del Colegio de Profesores.

OECD (2004) Informe de educación chilena. Santiago de Chile: OECD/Ministerio de Educación.

Rappaport, D.; Benavente J.M. y Meller, P. (2004) Rankings de universidades chilenas según los ingresos de sus titulados. Banco Central de Chile, Documento de Trabajo $N^{\circ}$ 306. Disponible en http://www.bcentral. cl/esp/estpub/estudios/dtbc/pdf/dtbc306.pdf

Ruffinelli, A. y Sepúlveda, L. (2005) Sistematización de la oferta de programas especiales de pedagogía en educación básica en instituciones de educación superior chilenas. Estudio realizado para MINEDUC por el Centro de Investigación y Desarrollo de la Educación, CIDE.

Ruffinelli, A. y Rojas, M.T. (2007) Actualización de la oferta de programas especiales de pedagogía en educación básica en instituciones de educación superior chilenas. Estudio de actualización realizado para MINEDUC por el Centro de Investigación y Desarrollo de la Educación, CIDE. 
44 CíRCULO DE SEGMENTACiÓN DEL SISTEMA EDUCATIVO CHILENO - Andrea Ruffinelli, Alexis Guerrero

Ruffinelli, A. (2009) Destino laboral de los egresados de pedagogía en educación básica de instituciones de educación superior chilenas: un análisis de la segmentación del mercado. Investigación financiada por el Consejo Superior de Educación, Chile.

Recibido: 28 de mayo de 2009

Aceptado: 16 de octubre de 2009 